\title{
TRIBUTACIÓN Y PERICIA CONTABLE DE MYPES EN PROCESO DE LAVADO DE ACTIVOS 2018-2019
}

\section{TAXATION AND ACCOUNTING EXPERTISE OF MYPES IN THE MONEY LAUNDERING PROCESS 2018-2019}

\author{
Ronald Prado Ramos ${ }^{1 *}$ \\ pradounsch@gmail.com \\ ${ }^{1}$ Universidad Nacional de San Cristóbal de Huamanga, Huamanga, Perú \\ Correspondencia: Ronald Prado Ramos. Email: pradounsch@gmail.com
}

Recibido: 11.08.21 | Aprobado: 22.09.21

\section{RESUMEN}

Objetivo: Evidenciar la influencia de la tributación en la pericia contable de micros y pequeños empresarios en un proceso de lavado de activos Método: El estudio fue cuantitativo de tipo descriptivo y exploratorio; el estudio transeccional no experimental. Se efectuó una inspección de campo, con observaciones a los micros y pequeñas entidades empleando encuestas durante el periodo 2018-2019. Resultados: La tributación influye directamente en la pericia contable practicada a MYPES toda vez que la práctica pericial se determina de una contabilidad netamente para fines tributarios, con formulación de profesionales externos que contiene incoherencias e inexactitudes propias con las transacciones que practica su organización Conclusiones: Los datos obtenidos como producto del estudio han permitido establecer que las micro y pequeñas empresas quedan a merced de una Tributación que condiciona su contabilidad y por ende la pericia contable practicada debilitándose propia a ser desestimada por el juzgador.

Palabras Claves: Tributación, micro y pequeña empresa, pericia contable, lavado de activos.

\begin{abstract}
Objective: To demonstrate the influence of taxation on the accounting expertise of micro and small entrepreneurs in a process of money laundering. Method: The study was quantitative, descriptive and exploratory; the non-experimental transectional study. A field inspection was carried out, with observations of the micro and small entities using surveys during the 2018-2019 period. Results: Taxation directly influences the accounting expertise practiced by MYPES since the expert practice is determined from an accounting purely for tax purposes, with the formulation of external professionals that contains inconsistencies and inaccuracies with the transactions carried out by their organization Conclusions: The Data obtained as a result of the study have made it possible to establish that micro and small companies are at the mercy of a Taxation that conditions their accounting and therefore the accounting expertise practiced, weakening it, to be dismissed by the judge.
\end{abstract}

KeyWords: Taxation, micro and small business, accounting expertise, money laundering. 


\section{INTRODUCCIÓN}

Ferullo y Grecchi (2006) con referencia a la relación de la tributación en la contabilidad afirma: "La tributación y la contabilidad presentan una estrecha relación siendo la primera en consecuencia de la segunda como la renta, patrimonio, consumo, capacidad contributiva, hecho imponible” (p.73)

Como es de conocimiento que las contabilidades en las MYPES muestran las variaciones patrimoniales tanto respecto a sus usuarios que desean conocer la variación evolutiva patrimonial, bienes de terceros en poder de la MYPES, contingencias diversas, así como demostrar resultados de operaciones a lo largo del tiempo, que nos permita demostrar y sustentar la posición económica - financiera a lo largo de su vida útil. Es así donde la contabilidad como ciencia tiene un producto para la toma de decisiones, así como los recursos que utiliza y demanda la empresa y/o persona, cuyo producto son los Estados Financieros que son elaborados por el profesional contable en base a las Normas y Principios de contabilidad generalmente aceptados, ellos traducidos en el Perú en normas internacionales de contabilidad (NIC) y normas internacionales de información financiera (NIIF). Respecto a estas normas señala Chavez (2018) que:

Si bien las MYPES cobran importancia sobre estas normas contables que tiene como objetivo reflejar la verdadera realidad económica de una empresa sea esta Jurídica o como persona natural con negocio cobra limitante al verse condicionado en su aplicación con el tratamiento tributario sea estos de ingresos y/o gastos, entre otras operaciones. (p.6)

Debemos de recordar y recalcar que esta distorsión y preponderancia de las normas tributarias y/o fiscales frente a la normativa contable es consecuencia primordialmente a: Una fuerte preponderancia e interés de la tributación en la información financiera, un desconocimiento de la normativa contable (NIC, NIIF, principios contables) por parte de los hacedores de los estados financieros (contadores), un condicionamiento de los propietarios y/o representantes empresariales frente a los hacedores de los estados financieros (contadores), a fin de limitar la información financiera por el temor de pagar impuestos.

Por consiguiente, la preparación, elaboración y presentación de los estados financieros conlleva a tener una contabilidad hibrida, formulando una información que no es financiera ni fiscal, distorsionando en sí el verdadero objetivo de los Estados Financieros.

A lo que la tributación se sujeta tan solo en la determinación de adiciones y deducciones de carácter fiscal partiendo de base de un resultado contable, en consecuencia, en la determinación de una base imponible. Por lo que es cierto que no cabe duda que la tributación NO debería intervenir en la contabilidad y que se trata de estudios autónomos y que de ser el caso se debería de adaptar los registros contables a las pasibles connotaciones fiscales. Ello en la práctica carece de valor y diferenciación al adaptar la contabilidad de MYPES solo y netamente para fines fiscales desde sus transacciones iniciales limitando y sesgando a los verdaderos fines de la contabilidad por lo que su preparación, formulación está influenciada y hasta condicionada por disposiciones fiscales. Dado la incongruencia de tributarizar la contabilidad financiera, 
existen registros que por no tener la exigencia y/o formalismo fiscal (tributario) son parcialmente considerados o no considerados en su totalidad dentro de la contabilidad, toda vez que el empresariado tiene escaso conocimiento y/o encarga a terceros (contadores) el registro de operaciones que no demuestran y sustentan una variación patrimonial.

Por lo que en un examen sobre variación patrimonial no solo cuenta con elementos netamente tributarios, sino sobre la existencia referida a la variación evolutiva patrimonial, bienes de terceros en poder de la MYPES, contingencias diversas, sustento de resultados de operaciones a lo largo del tiempo, que nos permita demostrar y sustentar la posición económica - financiera a lo largo de su vida útil. Lo dicho concuerda con Ferullo y Grecchi (2006) quienes mencionan que:

A lo largo del presente trabajo fuimos identificando coincidencias y divergencias entre la contabilidad general y la contabilidad fiscal en dos impuestos relevantes de nuestro sistema tributario: Impuesto a las Ganancias e Impuesto al Valor Agregado y señalando la gran incidencia que tienen las normas tributarias en la confección de los balances generales de las pequeñas y medianas empresas. (p.25)

Operaciones de conversión y/o transferencia de costo de vida como modalidad más utilizada en los casos de lavado de activos que cuyo sustento no solo es deficiente en la contabilidad de MYPES, sino que su registro o no registro que deviene de condicionamiento fiscal tributario. Así señala Cabello Reiniso (2017) quien concluye:

Estas operaciones son en cierta manera actos de conversión y/o transferencia en propiedades, empresas, transferencias bancarias, operaciones de bolsa y en costo de vida. Este último incrementado en los últimos años en un $100 \%$ tendencia observada es siempre creciente, así observamos que de representar un $75 \%$ al año 2013, los años 2014, 2015 y 2016 evolucionan a 85.00\%, 87.50\% y 100.00\%, respectivamente, si algunos años no es el 100\%. (p.85)

Costo de vida representada en erogaciones referido a la subsistencia y manutención, adquisición primaria (vivienda, alimentación, vestido, transporte, canasta familiar) ellos traducidos en una adquisición y/o mejora de viviendas, alimentación y vestido excesivo, adquisición de vehículos de última gama, incremento de la canasta familiar básica de manera abrupta entre otros.

A modo de ejemplo podemos observar tanto en ingresos como costos, gastos y contratos con terceros sin la formalidad tributaria (boletas de venta, contratos internos, comodatos, cesiones de uso, etc.) que no son contabilizadas o son parcialmente contabilizadas y que sin duda su inexistencia denota vacíos y dudas que agravan la condición económica - financiera en un proceso de lavado de activos.

El Ministerio Público, en su labor de realizar actos de investigación en su afán de evidenciar el delito, así como todos los actuados que permitan asegurar la PRUEBA que asegure formular acusaciones de carácter fiscal. Utiliza como medio de prueba tipificado en nuestro Código Procesal Penal la pericia y en específica para este tipo de delitos la pericia contable que sirve de base para recapitular, interpretar, averiguar 
los diferentes mecanismos financieros de los que se sirven las organizaciones criminales para el encubrimiento de sus delitos (Lobo, 2017, p. 18)

Es necesario comprender que la pericia contable es necesaria a fin de dar a conocer al magistrado sobre conocimientos de carácter técnico, científico un hecho en controversia. Tomando el magistrado a consideración como medio de prueba ello tipificado bajo el Art. 262 de, capítulo VI de nuestro código procesal civil. "El Código Procesal Penal establece lo siguiente: El examen en la audiencia se orientará a obtener una explicación sobre la comprobación que se efectuó respecto al objeto de la pericia, fundamentos y la conclusión que sostiene” (Ministerio de Justicia, 2004, art. 181).

La pericia contable como medio de prueba dentro de un proceso de lavado de activos debe de generar seguridad y mostrar un grado de certeza y ser debidamente probado contando para ello la información innegable de los libros y/o registros de contabilidad. Toda vez que sea admitida como prueba, será valorada por el juzgador.

Asimismo, la pericia contable no puede ser cuestionada sin datos contables, toda vez que no se puede cuestionar la pericia contable tan solo por afirmaciones de hecho que limiten negar la incriminación de hechos. Por ende, toda pericia contable sin un sustento contable carece de validez y genera dudas ante un proceso de lavado de activos pudiendo el juzgador desestimar dicha pericia. Lo dicho concuerda con Alvarado (2018) mencionando que:

La pericia contable, que se practica sobre hechos probados como los documentos tributarios, no es suficiente para comprender hechos que han sucedido en la vida real como el desarrollo de actividades informales (sin ser ilícitas), que, no habiendo sido incorporados al Expediente como tal, requieren de un estudio pormenorizado para llegar a entender sus causas, características y efectos. Esto solo puede ser posible utilizando en el campo del derecho a las ciencias económicas y financieras cuyos métodos reconstruyen con mayor eficacia y eficiencia actividades más allá de hechos meramente contables. (p.26)

Por lo que la doctrina contable (principios contables, NIIF, NIC) sirve de soporte en la pericia practicada toda vez que suman conocimientos técnicos y científicos que explican las diferentes variaciones patrimoniales de una persona natural y/o jurídica que explican y dan luces al magistrado o juzgador. A modo de conclusión, Morales (2009) manifiesta que:

La doctrina contable, es el soporte teórico del peritaje contable judicial en el Perú. Son los conocimientos sobre: a) La Contabilidad: Principios de Contabilidad Generalmente Aceptados y Normas Internacionales de Contabilidad y las Finanzas; b) Aspectos específicos de la Administración, la Economía, leyes sectoriales y específicas. Que los peritos contables desarrollan como sustento del examen pericial y sus resultados. Tienen carácter de criterios científicos y técnicos, en los que se apoyan los peritos contables para explicar los procedimientos y técnicas utilizados y sustentar los resultados o conclusiones del informe y/o dictamen pericial u opinión pericial solicitada por los jueces y fiscales de los fueros: civil, laboral y penal. Clasificados en soportes teóricos: a) Contables; b) Extracontables. (p.215) 
Bajo ese panorama la importancia que recoge la pericia contable ha dejado a la interpretación del juzgado en dar valor con base de dar seguridad jurídica en un proceso delictivo. En ese orden de ideas la presente investigación tiene por finalidad desarrollar indiscutiblemente la importancia de la prueba pericial contable al ser reemplazado la contabilidad comercial con una fiscal y está carecer de veracidad, probidad, que permita dilucidar las diversas cuestiones que se derivan de su práctica, así como servir de prueba valorada por cualquier acusación de carácter fiscal. Lo dicho guarda relación con los resultados recabados por Abad y Rodríguez (2019) quienes mencionan que:

Determinamos que el peritaje contable incide en los casos de lavado de activos, ya que es una herramienta contundente en los procesos judiciales, dicho esto se recomienda sensibilizar a los contadores públicos para especializarse como peritos, ya que esto permite contrarrestar los actos ilícitos como el de lavado de activos, el contador público debe tener un compromiso de responsabilidad social y que a través de su profesionalismo pueda reducir la corrupción y la delincuencia en nuestro país. (p.56)

\section{MATERIALES Y MÉTODOS}

La presente investigación fue descriptiva y exploratoria, de diseño no experimental. Para el estudio, se tiene una población 297 MYPES que están siendo procesadas bajo la investigación de lavado de activos, según para la selección de muestra se utilizó la siguiente fórmula 1:

\section{Fórmula 1}

$$
N=\frac{N Z^{2} a * p q}{(N-1) E^{2}+Z^{2} a+P Q}
$$

Donde; $Z=1.96$, valor para un nivel de confianza del 95\%, $N=297, p=50 \%$, $q=1$ $0.5, \mathrm{e}=1 \%$, Reemplazando valores tenemos:

$$
n=\frac{(297)(1.96)^{2}(0.50)(0.50)}{(297)(0.09)^{2}(0.50)(0.50)}
$$

$$
n=65
$$

El tamaño de la muestra es equivalente a 65 micro y pequeñas empresas a las cuales se les aplicó una encuesta que cursan un proceso preliminar en fase de investigación de lavado de activos ubicados en la ciudad de Ayacucho, del despacho de la fiscalía especializada en lavado de activos y pérdida de dominio del Distrito Fiscal de Ayacucho. El rumbo del estudio para la investigación fue de tipo cuantitativo con la intención de efectuar la recolección de datos con base a la medición aritmética y de interpretaciones estadísticos que permita entender la situación de las micro y pequeñas entes, para ello, se manejaron otras reglas equivalentes como la investigación de campo, a través de visitas a empresas; Asimismo se ha determinado la prospección histórica, con la cual se obtuvo documentos eficaces y de la periodicidad de operatividad." 


\section{RESULTADO}

De acuerdo con las variables de la investigación, se obtuvo establecer la influencia de la tributación en la prueba pericial contable de micros y pequeños empresarios en un proceso de lavado de activos, dando como resultado:

\section{Figura 1:}

\section{Contabilidad Netamente Tributaria}

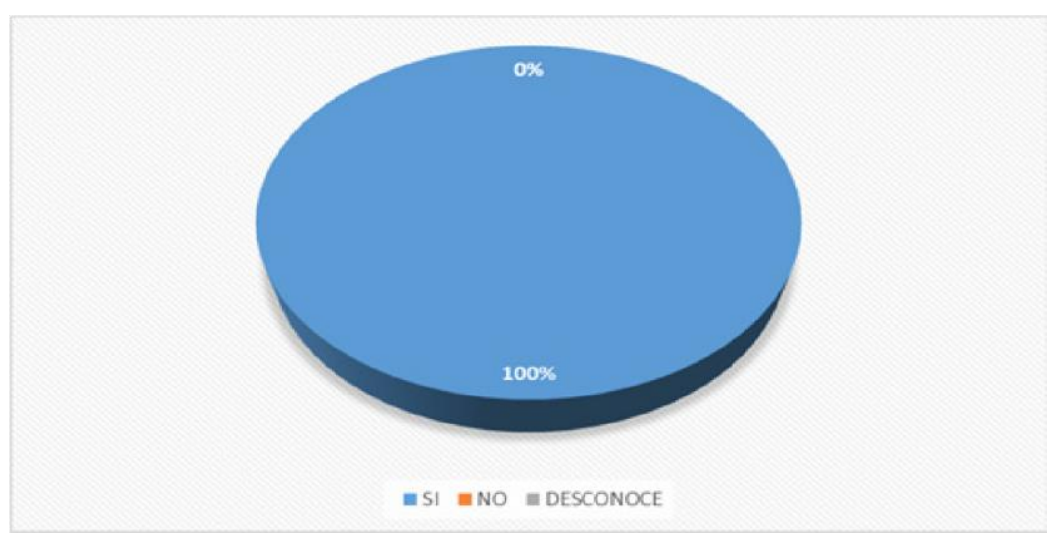

En la figura 1, se muestra que del total de micro y pequeñas empresas encuestadas entre propietarios y/o representantes legales el 100\% mencionan que la contabilidad que tienen dentro de sus organizaciones durante el periodo en estudio 2018 - 2019 es netamente para fines tributarios. Cabe indicar que los fines tributarios corresponden a actuaciones y/o acciones de cumplimiento sustancial y formal demandados por la Superintendencia Nacional de Aduanas y Administración tributaria (SUNAT).

Asimismo, se muestra en la figura 2 que del total de micro y pequeñas empresas encuestadas entre propietarios y/o representantes legales la contabilidad que tienen dentro de su organización lo llevan profesionales contables de manera externa (92\%) sin el conocimiento íntegro de transacciones, así como un limitado conocimiento de la organización, sus operaciones, socios, patrimonios, entre otros frente a un $8 \%$ que si tiene una contabilidad interna in situ que puede contrastar las operaciones y transacciones a ser contabilizadas así como rastrear su evolución y origen.

\section{Figura 2}

Tipo de Contabilidad que se lleva dentro de su organización

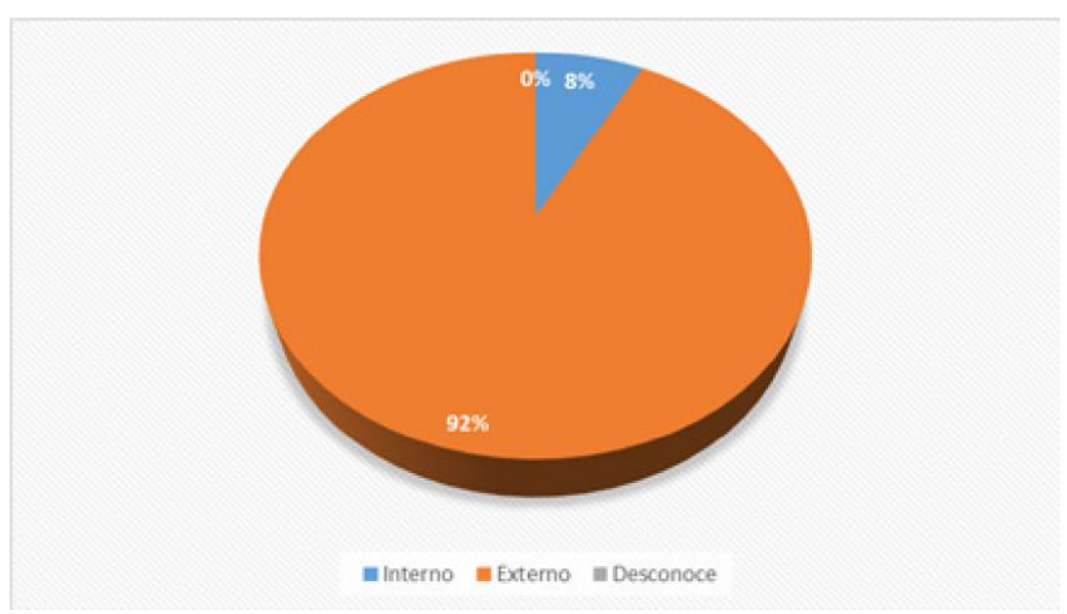


Del mismo modo se muestra en la figura 3 que del total de micro y pequeñas empresas encuestadas entre propietarios y/o representantes legales un 94\% menciona que no se contrata ni se lleva una contabilidad financiera integral interna por ser costosa y por no asumir la carga de trabajo. Frente a un $6 \%$ que no presta importancia a la contratación de una contabilidad financiera integral interna.

\section{Figura 3}

Razones porque no se lleva una contabilidad integral interna.

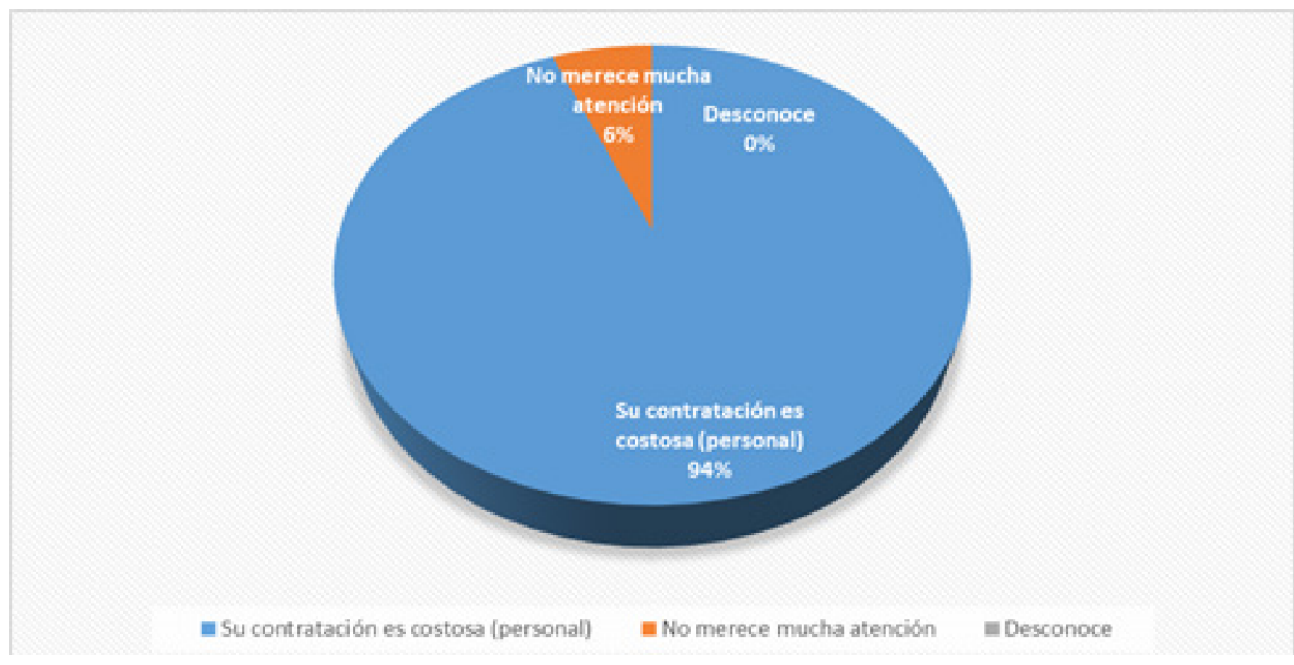

La contabilidad en Latinoamérica se ha traducido en el hecho de atribuir como Base hasta fuente de registro, clasificación, preparación y por no decir creación de políticas contables la normativa tributaria o fiscal, para la consecución y presentación de los Estados Financieros siguiendo erróneamente el esquema denotado en la Figura 4.

\section{Figura 4}

Distorsión histórica en la preparación de Estados Financieros

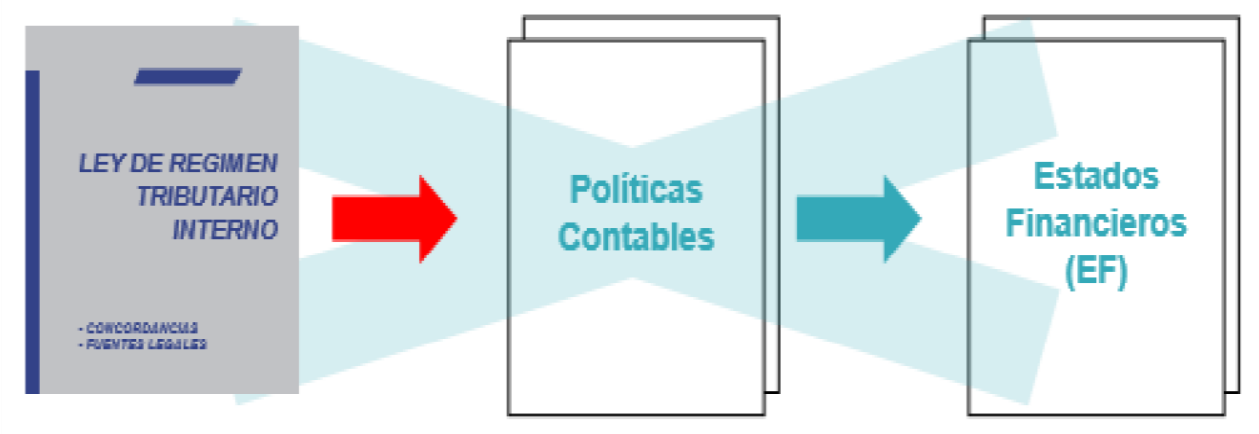

Fuente: (Chavez, 2018, pág. 11)

Asimismo, se muestra en la figura 5 que del total de micro y pequeñas empresas encuestadas entre propietarios o representantes legales un $77 \%$ de los empresarios tienen escaso conocimiento histórico de los incrementos patrimoniales que ha desarrollado como persona natural y como empresa. Así como un 15\% que tiene un conocimiento parcial. Lo que muestra la falta de interés del empresariado a contratar los servicios profesionales contables y por ello su falta y limitado conocimiento evolutivo patrimonial tanto de los intervinientes, así como de sus organizaciones. 


\section{Figura 5}

Conocimiento histórico de incrementos patrimoniales dentro de su organización

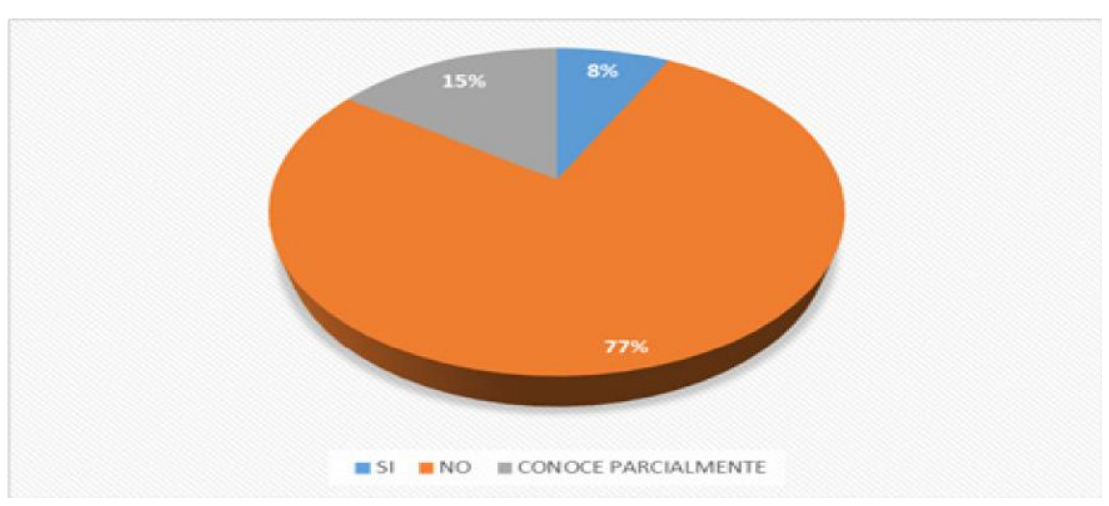

De otra manera en la figura 6 muestra que del total de micro y pequeñas empresas encuestadas entre propietarios o representantes legales un $95 \%$ presenta incoherencias e inexactitudes entre la pericia contable practicada y las transacciones financieras que realiza su empresa. Incoherencias e inexactitudes propias de operaciones no contabilizadas o parcialmente contabilizadas que escapan y sesgan las verdaderas operaciones y transacciones empresariales.

\section{Figura 6}

Incoherencias e inexactitudes encontradas en la pericia contable y transacciones que realiza su empresa.

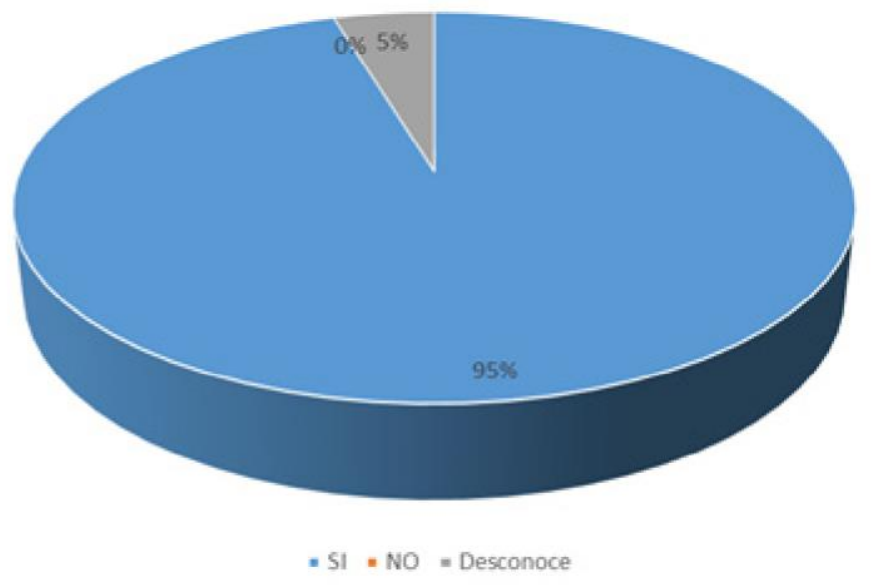

Los entrevistados, tienen claro que de conocer la situación actual por la que atraviesan no quedaría duda de contratar y realizar una contabilidad financiera integral que refleje el verdadero incremento patrimonial, así como las transacciones reales que realiza su empresa.

Asimismo, denotar que la pericia contable establece garantías de tipo competencial, imparcial, normativa. Teniendo como objeto formar parte de la base al planteamiento de una determinada hipótesis considerada por el magistrado. Así como válida tanto como prueba documental como testimonial. Siguiendo su importancia en el esquema denotado en la Figura 7.

Bajo este enfoque queda claro que la pericia contable practicada ofrece garantías tanto profesionales, procedimental propio de juicios técnicos y científicos capaz de 
asegurar una percepción y a ser considerado por el juzgador con mayor énfasis a una prueba documental.

Así la pericia contable como medio de prueba ante cualquier proceso tiene un objetivo importante el cual es de dar claridad técnica, científica y procesal probatoria al juzgador y/o magistrado.

\section{Figura 7}

Importancia de la pericia como medio probatorio
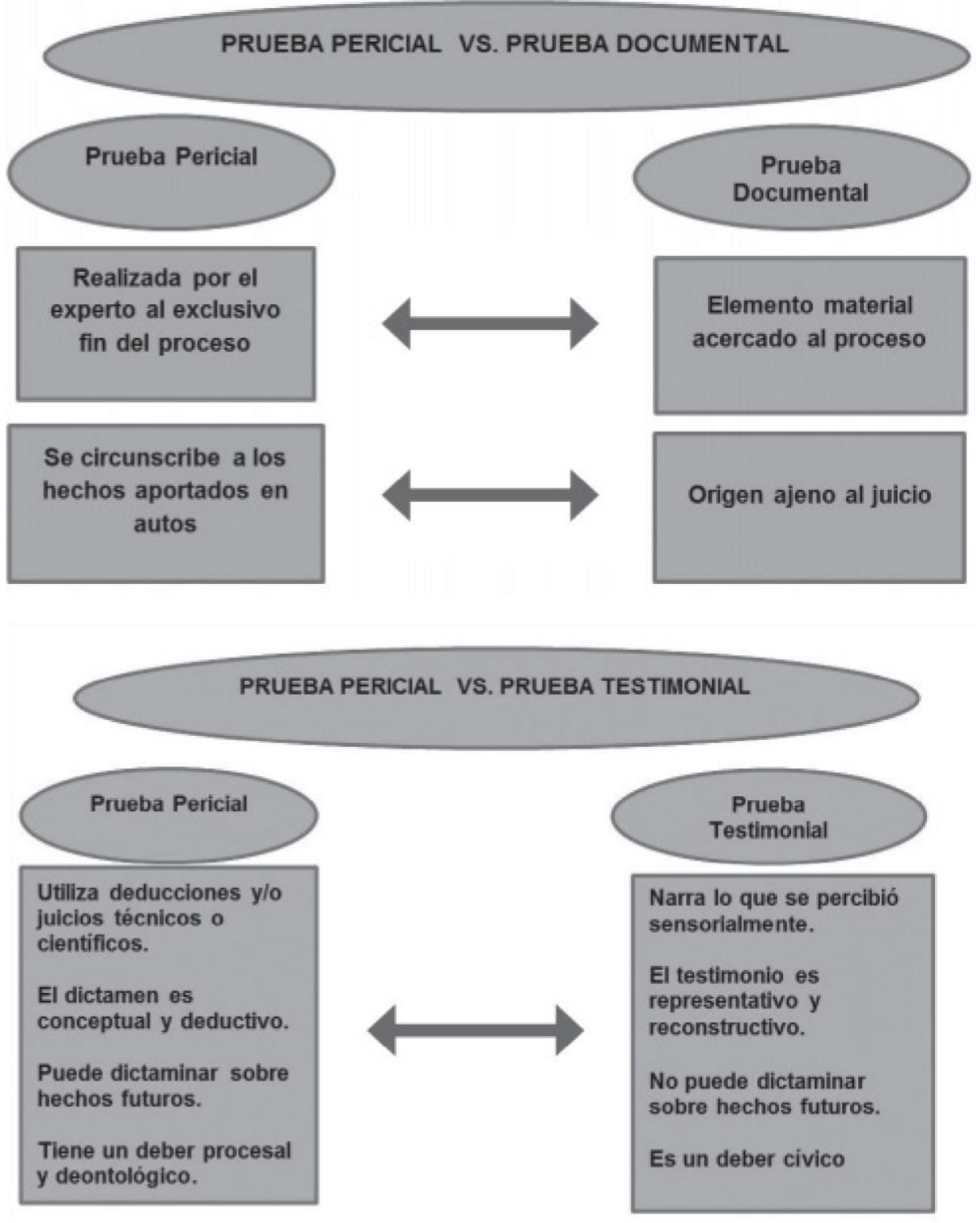

Fuente: (Ramon Ruffner de Vega, 2014, pág. 139)

\section{CONCLUSIONES}

Como el objetivo del presente artículo es evidenciar la influencia de la tributación en la pericia contable de micros y pequeños empresarios (MYPES) en un proceso de lavado de activos 2018-2019. Dentro de los resultados obtenidos en las figuras 1, 2 y 3 muestran que la contabilidad manejada en las micro y pequeñas empresas son netamente 
para fines tributarios, con formulación de profesionales (contadores) externos, contratados bajo esa modalidad por ser costosa y no asumir costos laborales. Asimismo, el resultado obtenido en la figura 5 evidencia que los micros y pequeños empresarios no cuentan con registros históricos sobre incrementos patrimoniales de sus organizaciones, vale decir realizar un estado financiero delineado para fines netamente tributarios. Otro si el resultado obtenido en las figuras 6 evidencia que al ser practicada una pericia contable dentro de un proceso de lavado de activos esta contiene incoherencias e inexactitudes propias con las transacciones que practica su organización. En síntesis, la influencia de la tributación en la pericia contable de micros y pequeños empresarios (MYPES) en un proceso de lavado de activos es condicionante, puesto que la contabilidad financiera practicada pierde el verdadero objetivo de los Estados Financieros. Así en un proceso de lavado de activos, la prueba pericial contable practicada queda debilitada y sin sustento por no coincidir con muchas de las transacciones acordes a la realidad económica financiera de la entidad y propia a ser desestimada por el juzgador.

\section{ORCID}

Ronald Prado Ramos: Universidad Nacional de San Cristóbal de Huamanga, Huamanga, Perú

\section{FUENTE DE FINANCIAMIENTO}

El estudio fue autofinanciado.

\section{CONFLICTOS DE INTERÉS}

El autor declara que no existe conflicto de interés.

\section{AGRADECIMIENTO}

No aplica.

\section{PROCESO DE REVISIÓN}

Este estudio ha sido revisado por pares externos en modalidad de doble ciego.

\section{DECLARACIÓN DE DISPONIBILIDAD DE DATOS}

No aplica.

\section{REFERENCIA BIBLIOGRÁFICA.}

Abad Valdivia, M. F., y Rodríguez Morales, E. M. (2019). El Peritaje contable en los procesos judiciales y su incidencia en la. Lima. https://repositorio.ucv.edu.pe/ bitstream/handle/20.500.12692/46001/Abad_VMF-Rodr\%C3\%ADguez_MEMSD. pdf?sequence $=1$ \&isAllowed $=\mathrm{y}$

Alvarado, E. M. (2018). Enfoques epistemológicos de la prueba pericial en los procesos judiciales por lavado de activos en la sala penal nacional. Tacna. http://repositorio.upt.edu.pe/bitstream/UPT/1411/1/Cordova-AlvaradoEduar.pdf

Cabello Reiniso, V. (2017). Peritaje Financiero vinculada al delito de lavado de activos en la sala penal nacional, 2013-2016 (Tesis para Optar Grado de Maestro en Gestión Pública). 
https://repositorio.ucv.edu.pe/bitstream/handle/20.500.12692/5415/Cabello_R VR.pdf?sequence $=1 \&$ is Allowed $=\mathrm{y}$

Chavez, L. A. (2018). NIIF y Tributación. Revista de Administración Tribuitaria CIAT, 1-5. Obtenido de https://www.ciat.org/Biblioteca/Revista/Revista_43/ espanol/2018_RA_43_chavez.pdf

Grecchi, A. M., y Ferullo, C. F. (2006). La contabilidad como Base de determinación Tributaria. Quintas Jornada Investigaciones en Facultad Universidad de Rosario, 73.https://core.ac.uk/download/pdf/90646641.pdf

Lobo, E. (17 de 10 de 2017). Algunas cuestiones sobre la prueba pericial contable en el proceso penal peruano. (U. S. Porres, Editor) https://derecho.usmp.edu.pe/ sapere/ediciones/edicion_11/concursos/2014/3ER_PUESTO.pdf

Ministerio de Justicia. (s.f.). Nuevo Código Procesal Penal. Decreto Legislativo No 957. Perù: El Diario el Peruano (29 de julio de 2004).

Morales, A. A. (2009). Enfoque doctrinario y factual del peritaje judicial contable en el Perú (2004-2008). Lima: Universidad Nacional Mayor de San Marcos. http:// cybertesis.unmsm.edu.pe/bitstream/handle/20.500.12672/4566/Arroyo_ma.pdf? sequence $=1$ \&is Allowed $=\mathrm{y}$

Ramon Ruffner de Vega, G. (2014). La Prueba Pericial. Revista de la Facultad de Ciencias Contables Quipukamayoc, 22, 139. https://revistasinvestigacion.unmsm.edu.pe/ index.php/quipu/article/view/11056/9935 